\title{
What can early Canadian experience screening for COVID-19 teach us about how to prepare for a pandemic?
}

\author{
Molly Lin MD, Alina Beliavsky MD, Kevin Katz MD CM MSc, Jeff E. Powis MD MSc, Wil Ng MHSc, \\ Victoria Williams MPH, Michelle Science MD MSc, Helen Groves MB BCh BAO PhD, Mathew P. Muller MD PhD, \\ Alon Vaisman MD MAS, Susy Hota MD MSc, Jennie Johnstone MD PhD, Jerome A. Leis MD MSc
}

- Cite as: CMAJ 2020 March 23;192:E314-8. doi: 10.1503/cmaj.200305; early-released March 6, 2020

Infographic available at www.cmaj.ca/lookup/suppl/doi:10.1503/cmaj.200305/-/DC1

A novel coronavirus (coronavirus disease 2019 [COVID-19]) emerged in Wuhan, China, in December 2019 and is considered a public health threat of international concern. As of Mar. 2, 2020, there were 90284 confirmed cases worldwide, with 80026 (89\%) cases and 2912 deaths identified in China, and another 10258 cases identified in 59 other countries, of which there were 27 confirmed in Canada. ${ }^{1}$ As of Feb. 21 in Ontario alone, 479 persons under investigation (PUI) for COVID-19 had been tested, 3 of whom tested positive.

Over the past few weeks, the epidemic has not slowed down, and there is growing concern for a COVID-19 pandemic, which would strain Canadian health care resources substantially. Prompt analytic description of the recent burden of screening and testing for COVID-19 on the health care system could identify challenges that should be addressed in the development of more effective policies and practices for health care delivery across sectors and jurisdictions before a pandemic emerges.

We present an analysis of data on PUI encountered over a 1-month period across 8 emergency departments in Toronto, Ontario, and discuss some of the potential drivers contributing to emergency department visits, to inform policies for future appropriate handling of the COVID-19 outbreak. As we continue to learn about the clinical presentation of COVID-19, international evidence suggests that most patients have mild cases, are not acutely ill and do not require hospital admission. ${ }^{2}$ This is confirmed by our own limited data and suggests that many emergency department visits may be avoidable.

\section{What have we learned from patients investigated for COVID-19 so far?}

Infection prevention and control (IPAC) teams at hospitals in the Greater Toronto Area are mandated to collect infectious disease surveillance data. We looked at data collected prospectively from Jan. 20 to Feb. 19, 2020, at 8 hospitals on patients who were

\section{KEY POINTS}

- A pandemic of coronavirus disease 2019 (COVID-19) is expected, which will lead to a surge of people who must be tested and treated.

- Most people investigated for COVID-19 are now assessed in the emergency department; they have mild illness, suggesting that these hospital visits may be avoidable.

- Barriers to assessment and testing for COVID-19 outside of acute care hospitals are likely contributing to these avoidable emergency department visits.

- Addressing barriers to community-based assessment and testing for COVID-19 is urgently needed to minimize emergency department overcrowding, infection of health care workers and risks to other acutely ill patients.

investigated for COVID-19; these anonymized data included date of assessment, demographic characteristics (age and sex), clinical information, epidemiologic risk factors, laboratory and radiographic results, COVID-19 test results, and whether the patient was admitted or discharged home.

We identified 135 patients who were tested for COVID-19; they represented $28 \%$ of the patients tested within Ontario in that period. Figure 1 depicts the geographic distribution of these PUI across our network of hospitals. The characteristics of these patients are described in Table 1 . The median age was 28 (interquartile range 23 to 49 ; range $<1$ to 88 ) years, and $95 \%$ presented to the emergency department without a need for emergency medical services. The most common presenting symptoms were cough or shortness of breath (82\%), subjective fever or measured temperature of $38.0^{\circ} \mathrm{C}$ or greater $(48 \%)$, and sore throat $(30 \%)$. Only 3 patients $(2 \%)$ required supplemental oxygen during their emergency department visit, and only $5 \%$ of patients were admitted to hospital, while all others were discharged with home isolation pending COVID-19 test results, under the supervision of the local public health unit. 


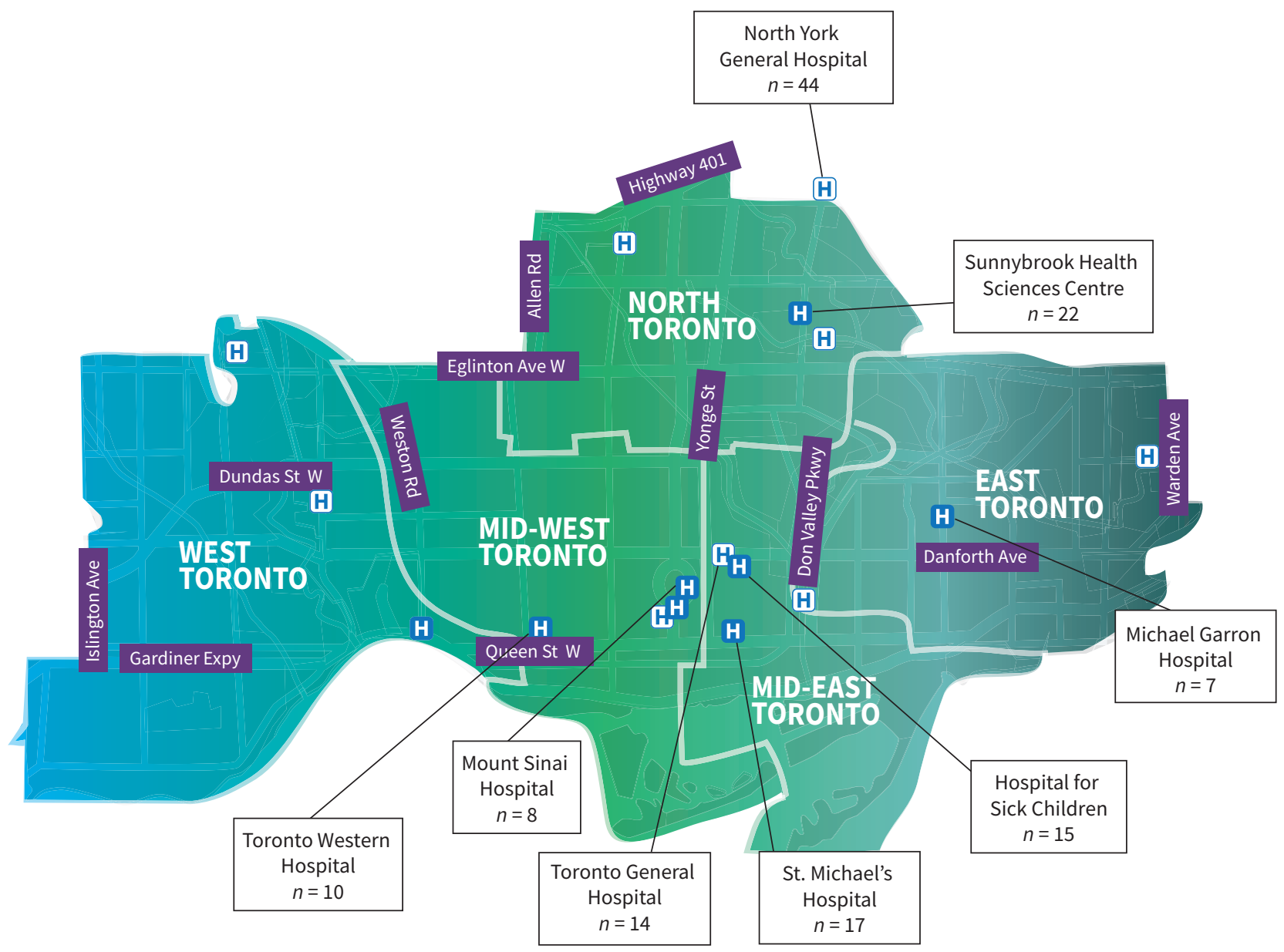

Figure 1: Distribution of person-of-interest cases across 8 hospitals in Toronto, Ontario $(n=135)$. Modified, with permission, from Toronto Central Local Health Integration Network.

In total, $111(82 \%)$ of the patients who underwent COVID-19 testing met formal provincial case definition for PUI as detailed on Feb. 7, 2020: a person with fever and/or onset of cough or difficulty breathing and relevant exposure (travel to mainland China in the 14 days before illness onset, close contact with a confirmed or probable case of COVID-19, or close contact with a person with acute respiratory illness who had been to mainland China within 14 days before their illness onset). ${ }^{3}$ In the context of evolving surveillance practices during the study period, $8 \%$ of those tested at our hospitals had returned from countries other than China, as many countries beyond China had reported cases that were not linked to travel from China. ${ }^{1}$ An additional $10 \%$ of people were tested despite having neither travelled outside of Canada nor had close contact with a person with probable or confirmed COVID-19 infection, which may reflect some people seeking reassurance that they did not have COVID-19. In 2 of the 8 hospitals, an additional 74 patients were assessed as possible PUI during the same period, but were ultimately not tested because they did not meet the case definition. As such, we estimate the total number of emergency department visits for assessment of PUI across our hospitals to be at least $40 \%$ higher than what we have reported.
The 135 patients whose data we analyzed are similar in age and sex distribution to those PUI recently described in the United States. ${ }^{4}$ In comparison to the largest case series of confirmed COVID-19 patients in China, where the median age was much older (56 yr), signs and symptoms and admission rate (5\%) were similar to those of the PUI assessed across our Toronto hospitals. ${ }^{5}$

\section{Why do people go to the emergency department for suspected COVID-19?}

To consider whether emergency department attendance by those with potential COVID-19 could be minimized, we need to understand why patients present to the emergency department.

First, provincial guidance in Ontario as of Jan. 23, 2020, and onward recommended the use of fit-tested N95 respirators in addition to droplet-contact precautions and airborne infection isolation rooms when possible for the assessment of PUI. ${ }^{6}$ This recommendation was based on the precautionary principle, as described in the Campbell report, ${ }^{7}$ which is intended for use when uncertainty exists with regard to the pathogen or the mode of transmission. The provincially recommended personal 
Table 1: Characteristics of 135 patients assessed between Jan. 20 and Feb. 19, 2020, across

8 emergency departments in Toronto, Ontario, and tested for COVID-19

\section{Characteristic}

Demographic characteristics

Age, yr, median (IQR)

Sex, male

Walked in $\dagger$

Clinical features

Subjective or measured fever $\left(\geq 38.0^{\circ} \mathrm{C}\right)$

Cough or shortness of breath

Sore throat

Gastrointestinal symptoms

Malaise/fatigue

Epidemiologic risk category

Travel from China $<14 \mathrm{~d}$

Close contact with probable or confirmed case of COVID-19

Close contact with person with respiratory symptoms who travelled to China $<14 \mathrm{~d}$

Travel from another Asian country $<14 \mathrm{~d}$

Supplemental oxygen required

Airborne-droplet-contact precautions

Results of investigations, median (IQR), $n=48$

White blood cell count, $\times 10^{9} / \mathrm{L}$

Neutrophils, $\times 10^{9} / \mathrm{L}$

Lymphocytes, $\times 10^{9} / \mathrm{L}$

Platelets, $\times 10^{\circ} / \mathrm{L}$

Chest radiography results

Pneumonia seen on chest radiograph

No pneumonia on chest radiograph

Chest radiography not done

Met provincial case definition for "person under investigation"3

COVID-19 confirmed

Disposition

Admitted to hospital

Discharged with home isolation and public health follow-up
No. $(\%)$ of patients ${ }^{\star}$

$28(23-49)$

128 (95)

$111(82)$

$40(30)$

$14(10)$

$23(17)$

$111(82)$

$19(14)$

$9(7)$

$11(8)$

$134(99)$

$7.3(6.1-10.0)$

$4.3(3.4-6.4)$

$2.0(1.1-2.3)$

$216(179-289)$

$9(7)$

$61(45)$

$111(82)$

$1(0.7)$

$128(95)$

Note: COVID-19 = coronavirus disease $2019, \mathrm{IQR}=$ interquartile range.

*Unless stated otherwise.

†Patient was not brought to emergency department by emergency medical services.

protective equipment (PPE) and infrastructure required to support airborne-droplet-contact precautions are not routinely available in most outpatient clinics, and this prevents the assessment and testing of low-acuity PUI in these settings. ${ }^{3}$ Although there is heterogeneity in the approach to recommended precautions in different jurisdictions nationally and internationally, to date, the World Health Organization (WHO) has not identified any evidence of airborne spread and thus recommends droplet-contact precautions unless an aerosolgenerating medical procedure is undertaken. ${ }^{8,9}$
Second, access to diagnostic testing for COVID-19 outside of acute care hospitals is limited during a time when testing PUI is critical to case identification for public health reasons. One barrier is primary care providers who are not prepared or equipped to collect nasopharyngeal or mid-turbinate specimens because in previous years they were discouraged from doing so for other viral respiratory infections, such as influenza. ${ }^{10}$

Third, most community-based providers do not have access to the same IPAC resources or expertise as acute care hospitals, such as readily available nasopharyngeal swabs and appropriate 
PPE. The uncertainty of a novel pathogen and the provincial guidance described above have presumably led providers and public health officials to refer patients to the emergency department at a higher rate than they would normally for patients with other viral respiratory syndromes.

Finally, Canadians' prior experience with severe acute respiratory syndrome (SARS) may drive emergency department visits. It is possible that many patients assume that hospital emergency departments are the best locations to be assessed for COVID-19. ${ }^{11}$

We acknowledge that these identified system drivers are speculative; further research is required to validate them.

\section{Why is it important to minimize the number of patients presenting to emergency departments for investigation?}

Recent data from Wuhan, China, show high rates of transmission of COVID-19 to health care workers (29\% in 1 series), which is higher than the rates seen with Middle East Respiratory Syndrome (MERS) and SARS (18.6\% and $21 \%$, respectively), highlighting a pressing need to contain mildly symptomatic patients who do not need acute medical services and to reduce unnecessary exposures to hospital staff and other patients. ${ }^{5,12,13}$ Systems that favour assessment of nearly all patients in hospital emergency departments will lead to a surge during a pandemic of COVID-19. Across our 8 hospitals, the number of airborne infection isolation rooms in the emergency department ranges from 2 to 16 , which must be reserved for patients requiring aerosolgenerating medical procedures. Further overcrowding of emergency departments with patients who are not so sick that they require management in hospital will lead to overcrowding into hallways, which will increase the risk of exposure and transmission and compromise access for patients with severe COVID-19 illness or other acute medical illnesses.

\section{How can we successfully reduce emergency department visits for suspected COVID-19?}

Recommendations for use of PPE in the community setting must be evidence based and clearly communicated to all health care workers, and a starting point could be the recommendations put forth by WHO for health care facilities. ${ }^{14}$ The approach of using droplet-contact precautions for patients who do not require aerosol-generating medical procedures is aligned with most available data. ${ }^{9,15,16}$

Using droplet-contact precautions would allow outpatient clinics to safely care for PUI and discharge them with homeisolation measures under the supervision of the local public health unit. This approach for patients who would otherwise not require hospital admission would prevent overcrowding in the emergency department and keep the number of exposures to a minimum. Equipping primary care physicians with the infrastructure to collect specimens and transport them to public health laboratories, or establishing COVID-19 assessment clinics in highrisk areas, will send the message to the public to go directly to these sites rather than to the emergency department.
Home testing of PUI is complementary to improving outpatient capacity and has been shown in a pilot study to reduce unnecessary ambulance use and hospital visits. ${ }^{17,18}$ This system, which is already in place for other communicable diseases such as tuberculosis, is likely being underused for COVID-19 PUI who are not acutely ill. ${ }^{19}$ Telehealth, which has been shown to be an effective way of detecting gastrointestinal infectious disease outbreaks in Ontario, could be expanded to promptly identify PUI and provide direct guidance to patients in the community without the need for an emergency department visit. ${ }^{20}$

Canada's public health infrastructure has come a long way since the days of the SARS outbreak in 2003, with a greater capacity to respond effectively to epidemics. ${ }^{21}$ Public health continues to play a pivotal role in close monitoring and isolation of PUI and confirmed cases, as well as contact tracing and active surveillance. ${ }^{22}$ They are also influential leaders in enhancing communication and providing guidance to the public, especially around when it is appropriate to seek acute medical care, and when it is appropriate to be assessed by community providers. ${ }^{22}$

\section{Conclusion}

The COVID-19 epidemic is evolving rapidly, and a pandemic appears imminent. Canada's preparedness for COVID-19 must extend beyond hospitals, as a surge of cases appears inevitable. Our early experience at 8 centres in Toronto shows that $95 \%$ of PUI are not acutely ill and do not require hospital admission. These unnecessary hospital visits may be driven by a lack of access to the recommended PPE and diagnostic testing, role uncertainty among community-based providers, and a lack of coordination between community providers and existing local public health units. These barriers need to be addressed urgently before a pandemic situation leads to emergency departments across the country being overwhelmed, with the knock-on effects of overcrowding, health care worker infection and risks to other acutely ill patients.

\section{References}

1. Coronavirus COVID-19 global cases by Johns Hopkins CSSE. Baltimore: Johns Hopkins University. Available: https://gisanddata.maps.arcgis.com/apps/ opsdashboard/index.html\#/bda7594740fd40299423467b48e9ecf6 (accessed 2020 Feb. 21).

2. Novel Coronavirus Pneumonia Emergency Response Epidemiology Team. The epidemiological characteristics of an outbreak of 2019 novel coronavirus diseases (COVID-19) [article in Chinese]. Zhonghua Liu Xing Bing Xue Za Zhi 2020;41:145-51 10.3760/cma.j.issn.0254-6450.2020.02.003.

3. COVID-19: guidance for the health sector; Toronto: Ministry of Health and Long-Term Care; modified 2020 Feb. 28. Available: www.health.gov.on.ca/en/ pro/programs/publichealth/coronavirus/2019_guidance.aspx (accessed 2020 Feb. 21).

4. Bajema KL, Oster AM, McGovern OL, et al.; 2019-nCoV Persons Under Investigation Team; 2019-CoV Persons Under Investigation Team. Persons evaluated for 2019 novel coronavirus - United States, January 2020. MMWR Morb Mortal Wkly Rep 2020;69:166-70.

5. Wang D, Hu B, Hu C, et al. Clinical characteristics of 138 hospitalized patients with 2019 novel coronavirus-infected pneumonia in Wuhan, China. JAMA 2020 Feb. 7 [Epub ahead of print]. doi: 10.1001/jama.2020.1585.

6. January 23, 2020 letter from $\mathrm{CMOH}$ Dr. Barbara Yaffe. Toronto: Ontario Hospital Association; 2020 Jan. 23. Available: www.oha.com/Bulletins/ACMOH $\% 20$ letter\%202020-01-23.pdf (accessed 2020 Mar. 2). 
7. The SARS Commission. Toronto: The Archives of Ontario; 2006. Available: www. archives.gov.on.ca/en/e_records/sars/report/index.html (accessed 2020 Feb. 3).

8. Novel coronavirus (COVID-19). Vancouver: BC Centre for Disease Control. Available: www.bccdc.ca/health-professionals/clinical-resources/novel-coronavirus -(covid-19) (accessed 2020 Feb. 23).

9. Q\&A on infection prevention and control for health care workers caring for patients with suspected or confirmed 2019-nCoV. Geneva: World Health Organization; 2020. Available: www.who.int/news-room/q-a-detail/q-a-on-infection-prevention-and -control-for-health-care-workers-caring-for-patients-with-suspected-or-confirmed -2019-ncov (accessed 2020 Feb. 21).

10. Test information index: respiratory viruses (including Influenza). Toronto: Public Health Ontario. Available: www.publichealthontario.ca/en/laboratory-services/ test-information-index/virus-respiratory (accessed 2020 Feb. 23).

11. Peeri NC, Shrestha N, Rahman MS, et al. The SARS, MERS and novel coronavirus (COVID-19) epidemics, the newest and biggest global health threats: What lessons have we learned? Int. J Epidemiol 2020 Feb. 22 [Epub ahead of print]. doi: 10.1093/ije/dyaa033.

12. Park JE, Jung S, Kim A, et al. MERS transmission and risk factors: a systematic review. BMC Public Health 2018;18:574.

13. Malave A, Elamin EM. Severe acute respiratory syndrome (SARS): lessons for future pandemics. Virtual Mentor 2010;12:719-25.

14. The COVID-19 risk communication package for healthcare facilities. Geneva: World Health Organization; 2020. Available: https://iris.wpro.who.int/ bitstream/handle/10665.1/14482/COVID-19-022020.pdf (accessed 2020 Mar. 2).
15. Infection prevention and control during health care when novel coronavirus (nCoV) infection is suspected. Geneva: World Health Organization; 2020. Available: www. who.int/publications-detail/infection-prevention-and-control-during-health-care -when-novel-coronavirus-(ncov)-infection-is-suspected-20200125 (accessed 2020 Feb. 23).

16. Coronavirus disease (COVID-19): outbreak update. Ottawa: Public Health Agency of Canada. Available: www.canada.ca/en/public-health/services/ diseases/2019-novel-coronavirus-infection.html (accessed 2020 Feb. 23).

17. Mahase E. Coronavirus: home testing pilot launched in London to cut hospital visits and ambulance use. BMJ 2020;368:m621.

18. Silversides A. Canada's ability to respond to a national health crisis hampered by jurisdictional issues, untested emergency plans. CMAJ 2009;180:1193-4.

19. Tuberculosis program guideline, 2018. Toronto: Ministry of Health and Long-Term Care; 2018. Available: www.health.gov.on.ca/en/pro/programs/publichealth/ oph_standards/docs/protocols_guidelines/Tuberculosis_Program_Guideline _2018.pdf (accessed 2020 Mar. 2).

20. Caudle JM, van Dijk A, Rolland E, et al. Telehealth Ontario detection of gastrointestinal illness outbreaks. Can J Public Health 2009;100:253-7.

21. Wu Z, McGoogan JM. Characteristics of and important lessons from the coronavirus disease 2019 (COVID-19) outbreak in China: summary of a report of 72 314 cases from the Chinese Center for Disease Control and Prevention. JAMA 2020 Feb. 24 [Epub ahead of print]. doi: 10.1001/jama.2020.2648.

22. Heymann DL, Shindo N. WHO Scientific and Technical Advisory Group for Infectious Hazards. COVID-19: What is next for public health? Lancet 2020;395:542-5.
Competing interests: Jeff Powis reports grants from Gilead, outside of the submitted work. Helen Groves reports an honorarium received for presenting research at educational meeting sponsored by AbbVie, outside the submitted work. Susy Hota reports a grant from Finch Therapeutics, outside the submitted work. No other competing interests were declared.

This article has been peer reviewed.

Ethics approval: Research ethics board (REB) approval was sought at all 8 institutions; REB approval was granted by 3 institutions and waived at all remaining sites.

Affiliations: Department of Medicine (Lin, Beliavsky, Katz, Powis, Muller, Vaisman, Hota, Leis), University of Toronto; North York Gen- eral Hospital (Katz, Ng), Toronto, Ont.; Michael Garron Hospital (Powis), East York, Ont.; Sunnybrook Health Sciences Centre (Williams, Leis); Hospital for Sick Children (Science, Groves); Sinai Health System (Johnstone); Departments of Paediatrics (Science, Groves), and Laboratory Medicine and Pathobiology (Katz, Johnstone), University of Toronto; Unity Health Toronto (Muller); University Health Network (Vaisman, Hota), Toronto, Ont.

Contributors: Molly Lin and Alina Beliavsky contributed to the formal analysis and drafting of the manuscript. Jeff Powis, Kevin Katz, Alon Vaisman, Susy Hota, Michelle Science, Helen Groves, Mathew Muller and Jennie Johnstone contributed to the data curation, writing and review, and editing. Victoria Williams and Wil
$\mathrm{Ng}$ contributed to the data curation, methodology, writing and review, and editing. Jerome Leis contributed to the conceptualization, data curation, formal curation, formal analysis, supervision, writing and review, and editing. All authors gave final approval of the version to be published and agreed to be accountable for all aspects of the work.

Acknowledgements: The authors thank all infection prevention and control teams and clinicians involved in the surveillance and care of persons under investigation for coronavirus disease 2019. The authors thank Dr. Tia Pham for her review and comments on an earlier version of this manuscript.

Correspondence to: Jerome Leis, Jerome.Leis@sunnybrook.ca 